\title{
Streptobacillus felis, a member of the oropharynx microbiota of the Felidae, isolated from a tropical rusty-spotted cat
}

\author{
Ahmad Fawzy • Jörg Rau • Karin Riße • Nicole Schauerte $\cdot$ Christina Geiger • \\ Jochen Blom • Can Imirzalioglu • Jane Falgenhauer • Alexa Bach • \\ Christiane Herden - Tobias Eisenberg (i)
}

Received: 25 May 2020/Accepted: 22 July 2020/Published online: 9 August 2020

(C) The Author(s) 2020

\begin{abstract}
Streptobacillus felis is a fastidious microorganism and a novel member of the potentially zoonotic bacteria causing rat bite fever. Since its description, this is the second isolation of S. felis in a diseased member of the Felidae. Interestingly, the strain from this study was isolated from a zoo held, rusty-spotted cat (Prionailurus rubiginosus), with pneumonia, thereby indicating a possible broader host range in feline species. A recent preliminary sampling of domestic cats (Felis silvestris forma catus) revealed
\end{abstract}

Electronic supplementary material The online version of this article (https://doi.org/10.1007/s10482-020-01454-x) contains supplementary material, which is available to authorized users.

A. Fawzy

Faculty of Veterinary Medicine, Department of Medicine and Infectious Diseases, Cairo University, Cairo, Egypt

A. Fawzy $\cdot$ K. Riße $\cdot$ T. Eisenberg $(\bowtie)$

Department of Veterinary Medicine, Hessian State

Laboratory (LHL), Schubertstr. 60, 35392 Giessen,

Germany

e-mail: Tobias.Eisenberg@lhl.hessen.de

J. Rau

Chemical and Veterinary Analysis Agency Stuttgart, Schaflandstr. 3/2, 70736 Fellbach, Germany

N. Schauerte · C. Geiger

Frankfurt Zoo, Bernhard-Grzimek-Allee 1, 60316 Frankfurt, Germany that this microorganism is common in the oropharynx, suggesting that $S$. felis is a member of their normal microbiota. Due to unawareness, fastidiousness, antibiotic sensitivity and lack of diagnostics the role of S. felis as a cat and human pathogen might be underreported as with other Streptobacillus infections. More studies are necessary to elucidate the role of $S$. felis in domestic cats and other Felidae in order to better estimate its zoonotic potential.

Keywords Streptobacillus felis $\cdot$ Rat bite fever - Cat reservoir $\cdot$ Zoonosis $\cdot$ Immuno-histochemistry (IHC)

\footnotetext{
J. Blom

Bioinformatics and Systems Biology, Justus-Liebig-

University Giessen, Heinrich-Buff-Ring 58,

35392 Giessen, Germany

C. Imirzalioglu $\cdot$ J. Falgenhauer

Institute for Medical Microbiology, Justus Liebig University Giessen, Schubertstr. 81, 35392 Giessen, Germany

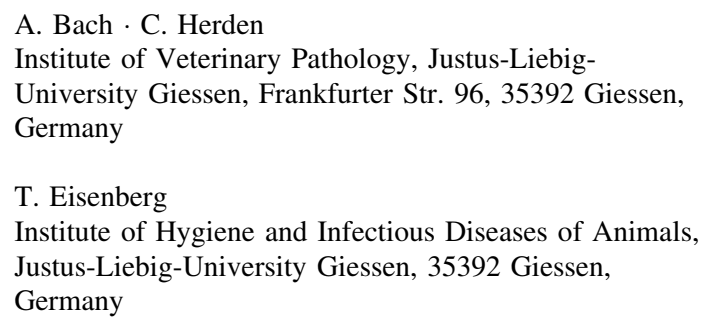




\section{Introduction}

Streptobacillus (S.) moniliformis (Leptotrichiaceae, Fusobacteriales) has been the longstanding unique species in this genus (Levaditi et al. 1925). This bacterium represents the most important causative microorganism of rat bite fever (RBF) and its foodborne variant, Haverhill fever (Eisenberg et al. 2018). RBF is typically characterized by a triad of fever, arthritis and a maculopapular, petechial or pustular rash, but severe causes of infection may include lifethreatening sequelae (Eisenberg 2017; Eisenberg et al. 2017a; Gaastra et al. 2009). A number of studies have stated a risk for RBF even through contacts to various non-rodent animal species like dogs, cats, weasels and ferrets as well as livestock animals. However, the proper identification of these microorganisms was not carried out and such isolates have not been stored. Recently, [S.] hongkongensis (Woo et al. 2014), S. felis (Eisenberg et al. 2014), S. notomytis (Eisenberg et al. 2015b), S. ratti (Eisenberg et al. 2016) and $S$. canis (Eisenberg et al. 2020b) were described as novel species. Whereas $S$. notomytis and $S$. ratti are closely associated with black rats (Rattus rattus), [S.] hongkongensis has exclusively been isolated from humans (Lau et al. 2016; Woo et al. 2014) and was recently found to belong to a novel genus, Pseudostreptobacillus (Eisenberg et al. 2020a). S. felis and $S$. canis were only once isolated from clinical disease in animals, i.e. from a cat with pneumonia and a dog with phlegmon, respectively (Eisenberg et al. 2015a, 2020b). However, with respect to zoonotic potential, $S$. notomytis has been found to also cause RBF in humans (Fukushima et al. 2017; Ogawa et al. 2018) and a similar case of RBF could recently be attributed to S. felis for the first time (Matt et al. 2020). Interestingly, various Streptobacillus phylotypes consistent with 16S rRNA gene sequence based operational taxonomic units (OTU) have been described from humans and various animal species (Fig. 1). We here report a second strain of $S$. felis, isolated from a tropical rusty-spotted cat (Prionailurus rubiginosus), one of the smallest members of Felidae, that succumbed to infection.

\section{Materials and methods}

Case description

A breeding group of the endangered rusty-spotted cat (Prionailurus rubiginosus phillipsi), a subspecies native to humid zones of Sri Lanka, is managed for ex situ breeding purposes in a German zoo. The cats have been bred in the same zoo or within the European studbook program and are housed individually or in breeding pairs. From the breeding group no significant morbidities and mortalities have occured, but individual animals have suffered from intermittant signs of kitty flu like sneezing, epiphora, elevated respiratory rate, reduced appetite, corneal ulceration in the years before this study. In the actual case, a female displayed bilateral blepharitis, weakness, respiratory distress and anorexia. Intra vitam tests for feline parvovirus, coronavirus and protozoa revealed negative results. Due to disease progression, the animal was euthanized.

Pathological investigation

A gross pathology examination and histology were performed. For histopathological examination, specimens of multiple organs were fixed in buffered $4 \%$ formalin, processed by standard methods and embedded in paraffin. Microtome sections were stained with hematoxylin-eosin (HE).

Immuno-histochemistry (IHC) for S. moniliformis

The IHC examination of the formalin fixed paraffin embedded (FFPE) samples taken for histopathological examination was performed using a recently established and not yet published protocol. Briefly, this protocol utilizes a standard IHC procedure with the use of heat induced antigen demasking in target retrieval solution (Dako Cytomation Denmark AS, Glostrup, Denmark), followed by goat serum (Life Technologies Corporation, Paisley, UK) and avidin/ biotin blocking agent (Linaris Biologische Produkte $\mathrm{GmbH}$, Dossenheim, Germany) in order to block nonspecific binding and reactions, respectively. The primary antibody used was an affinity purified polyclonal rabbit-anti-S. moniliformis antibody supplied by Davids Biotechnologie GmbH (Regensburg, Germany). A goat-anti-rabbit IgG biotinylated antibody 


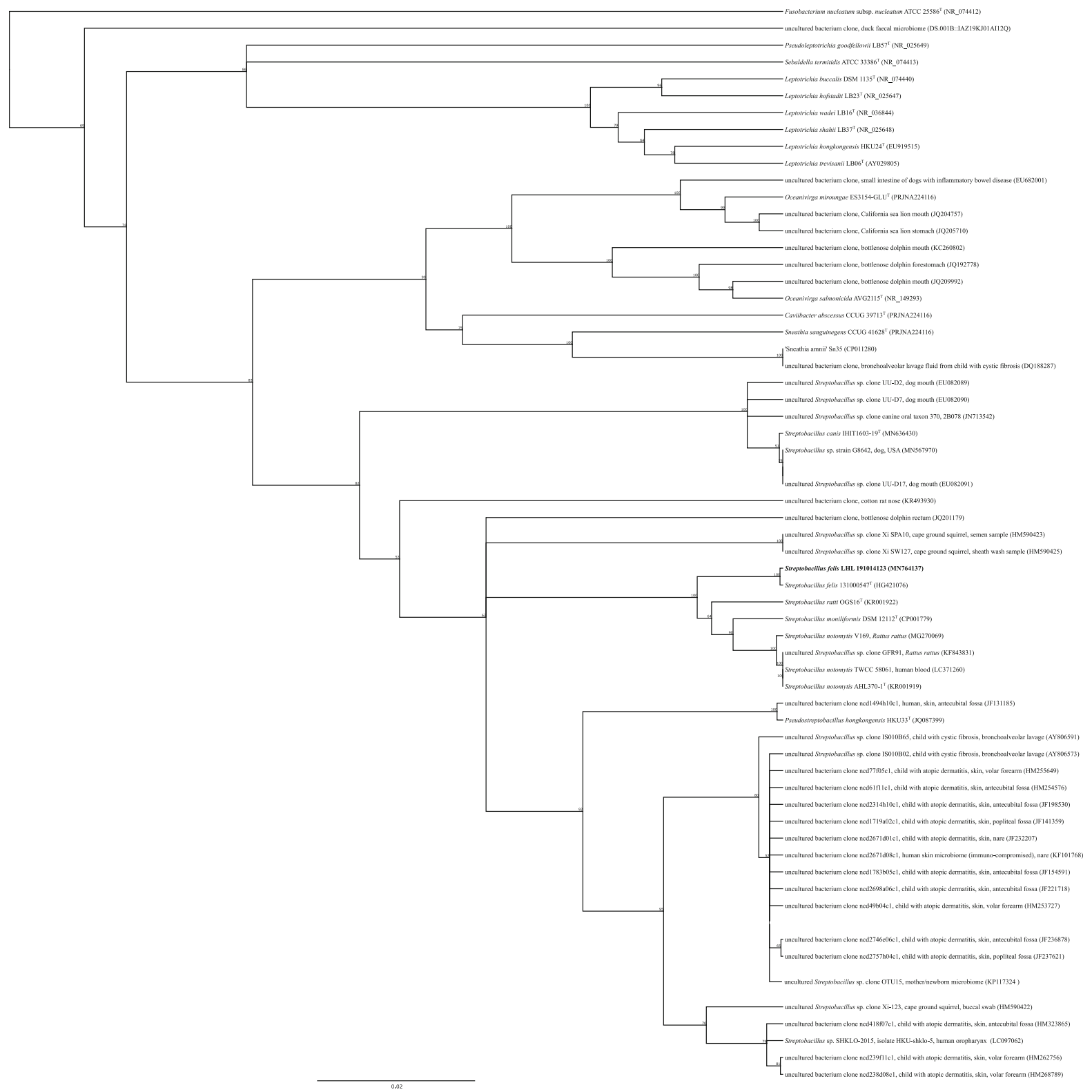

Fig. 1 UPGMA consensus tree depicting phylotypes and species of the family Leptotrichiaceae. The data set was based on 16S rRNA gene sequences and processed in Geneious vers. 8.1.9 (Kearse et al. 2012) using a Clustal W nucleotide alignment with standard settings and rapid bootstrap analysis

(Vector Laboratories, Burlingame, USA) served as a secondary antibody and allowed the detection of the antigen-antibody complex using the Vectastain ABCElite Kit (Linaris). Diaminobenzidine (DAB; SigmaAldrich Chemie GmbH, Steinheim, Germany) was added, resulting in a brown-colored precipitate forming where antibody have bound.
(1,000 bootstraps). GenBank accession numbers are given in parentheses. Numbers at branch nodes refer to bootstrap values; Fusobacterium nucleatum is used as outgroup. "T" indicating type strain; Bar, 0.02 nucleotide substitutions per site

FFPE samples of the lung of a C57BL/6 mouse that was experimentally infected with $S$. moniliformis (Fornefett et al. 2017) underwent the same protocol and served as positive controls. For a negative control, FFPE samples of the rusty-spotted cat underwent the described protocol with only the primary antibody being replaced with negative control rabbit 
immunoglobulin fraction (Dako). Evaluation of the immune-histochemical examination was performed using a transmission light microscope.

Phenotypic characterization

\section{Bacterial isolation and physiological properties}

Bacterial isolates were obtained and isolates were identified using standard microbiological examinations. Briefly, native tissue samples were processed for microbial culture by inoculating flame sterilized, freshly cut tissue surfaces onto culture media (Columbia agar with 5\% sheep blood [SBA; Oxoid, Wesel, Germany] and Gassner agar [VWR, Darmstadt, Germany]). Agar plates were incubated for up to $48 \mathrm{~h}$ at $20{ }^{\circ} \mathrm{C}$ using aerobic and microaerobic culture conditions. Phenotypic characterization of streptobacilli is known to yield only few weakly positive reactions (Eisenberg et al. 2015c), however, standard microbiological procedures included tests for hemolysis on SBA, catalase activity with $3 \% \mathrm{H}_{2} \mathrm{O}_{2}$ on microscopic slides and for presence of cytochrome oxidase with the BBL DrySlide ${ }^{\circledR}$ oxidase system (Becton-Dickinson, Heidelberg, Germany). Urease, hydrogen sulfide, indole, motility and oxidative and fermentative glucose assimilation were tested on Christensen agar, SIM and OF medium in slant agar tubes, respectively (all Merck, Darmstadt, Germany). Microscopic examinations of fixed smears were performed using Gram's stain. For further identification attempts, the Omnilog GEN III plate identification system (Biolog, Hayward, USA) was utilized for the first time using the most sensitive protocols for fastidious bacteria (C1 and C2) with and without addition of $10 \%$ bovine serum according to manufacturer's recommendations.

Matrix-assisted laser desorption/ionization-time of flight mass spectrometry (MALDI-TOF MS)

Mass spectrometry procedure has been recently described in detail (Eisenberg et al. 2018, 2020b). The commercial database used (DB 8,468; BrukerDaltonics) comprised 24 spectra each from $10 \mathrm{~S}$. moniliformis strains. Reference spectra from wellcharacterized, quality-controlled strains of all other Streptobacillus/Pseudostreptobacillus species and most other members of the Leptotrichiaceae were added to the database from previous studies
(Eisenberg et al. 2020b; Rau et al. 2016). Identification was done with the commercial Bruker database, and with the extended database.

Molecular characterization of isolate LHL191014123 obtained from liver tissue

PCR analysis Two earlier designed PCR assays for the detection of $S$. moniliformis were employed to detect characteristic amplicon sizes of approximately 269 and 1,190 bp also for the rusty-spotted cat strain LHL191014123 (primers S5: 5'-CAT ACT CGG AAT AAG ATG G-3' and AS2: 5'-GCT TAG CTC CTC TTT GTA C-3') (Kimura et al. 2008) and [primers SbmF: $5^{\prime}-\mathrm{GAG}$ AGA GCT TTG CAT CCT- $3^{\prime}$ and SbmR: 5'-GTA ACT TCA GGT GCA ACT-3'; Nicklas, cited in (Rohde et al. 2008)]. It was recently found that these PCR assays are rather genus than species specific (Eisenberg et al. 2015c). Therefore, we have recently designed primers (forward: $5^{\prime}$ - AGT ATG GGA AAT AGT AGA TAA TAG- $3^{\prime}$ and reverse $5^{\prime}$ - ACT GTA GAT TGT GAG TTC TT- $3^{\prime}$ ) that could specifically amplify a partial sequence of the $\operatorname{gyr} B$ gene (732 bp) of the $S$. felis genome (Matt et al. 2020). The PCR reaction components and cycling conditions were carried out as previously described in Fawzy et al. (2016) with minor modifications (annealing temperature was $53{ }^{\circ} \mathrm{C}$ and elongation time was $90 \mathrm{~s}$ ).

\section{Whole genome sequencing}

Whole genome sequencing (WGS) was carried out to get insight into a core genome based phylogeny and compare the rusty-spotted cat's strain with established type strain genomes from the same family. The genome sequence of strain LHL191014123 was generated by de-novo assembly with reads from Illumina technology. In brief, DNA was isolated from cells grown for 3 days at $37{ }^{\circ} \mathrm{C}$ on TSA supplemented with $20 \%$ horse serum using a PureLink genomic DNA kit (Thermo Fisher). The library was prepared with a Nextera XT library preparation kit (Illumina) and sequenced on NextSeq 500 (mid output kit v2, $2 \times 150$ bp) instruments. The genome assembly was carried out by SPAdes (version 3.10.1), resulting in 163 contigs with $179 \times$ average coverage.

Phylogenetic and phylogenomic analyses For a first phylogenetic placement, a tree based on nearly full- 
length 16S rRNA gene sequences was constructed with Geneious vers. 8.1.9 (Kearse et al. 2012) using a Clustal W nucleotide alignment with standard settings and a Neighbor-Joining (NJ) tree (data not shown). Therefore, the 16S rRNA gene sequences of all type strains of the Leptotrichiaceae were obtained from GenBank and for strain LHL191014123 deduced from the full genome sequence (s. below). For a more detailed view into the phylogenetic relationship of strain LHL191014123 and all other Streptobacillus species the criteria of Woo et al. (2014) were considered. Phylogenetic analyses based on near full-lengths nucleotide sequences of the groEL, gyrB and recA genes were performed for all Streptobacillus species and the type species of all other genera of the Leptotrichiaceae. Respective nucleotide sequences were aligned using ClustalW implemented in Geneious vers. 8.1.9 (Kearse et al. 2012) and visualized as unweighted pair group method with arithmetic mean (UPGMA) phylogenetic trees (based on 1,000 replications [bootstrap analysis]). A representative tree for the $\operatorname{gyr} B$ gene is shown in Suppl. Fig. S1. The average nucleotide identity (ANI) values and core genome phylogeny were calculated for strain LHL191014123 in comparison with type strain genomes of the family Leptotrichiaceae using the EDGAR 2.3 platform (Blom et al. 2016). ANI values were computed as described by Goris et al. (2007) and as implemented in JSpecies (Richter and Rossello-Mora 2009).

\section{Results}

Gross pathology

The rusty-spotted cat weighed approx. $1.9 \mathrm{~kg}$, which constitutes a normal weight of this small wildcat. The female was born in 2010 and was 9-years old at the time of death. During post mortem examination a light creamy fur, possibly indicating signs of chronic cat flu, was found in the left cavum nasi, accompanied by a light hemorrhagic exudate.

\section{Histo-pathology}

A moderate follicular hyperplasia was noted in the spleen. Focal edema and emphysema were found in histological sections of lung tissue. Focally, fibrin, desquamated alveolar macrophages and neutrophil granulocytes with occasional phagocytized bacteria were detected in bronchioles and pulmonary alveoli. Single cysts were found in the kidneys.

Immuno-histochemistry

The IHC examination using a method designed for the detection of S. moniliformis in tissue samples revealed negative results in all examined tissues of the rustyspotted cat. Positive and negative controls were successfully showing the expected results, reaffirming sufficient specificity for the detection of $S$. moniliformis.

General microbiology

Bacterial culture revealed growth of $S$. felis in all tissues, except intestine and intestinal lymph node. The semiquantitative number of streptobacilli as obtained by counting colonies on the directly inoculated agar surface was found to be low $(<20)$ in spleen and kidney, moderate (20-50) in liver and lung and high $(>50)$ in the nasal cavity.

Varied growths of other Gram-positive and Gramnegative microbiota were cultivated from other tissues and identified as Enterococcus faecalis, Vagococcus teuberi and Escherichia coli using MALDI-TOF MS. Selective verification procedures for purely microaerobic bacteria, Chlamydia spp., Mycoplasma spp. or Salmonella spp. and herpesviruses revealed negative results throughout (data not shown).

\section{Parasitology}

A coproscopy flotation technique was negative.

Phenotypic characterization of isolate

LHL191014123 obtained from liver tissue

\section{Biochemical identification}

Due to the fastidious growth, physiological reaction patterns are generally very weak in members of the genus Streptobacillus [Eisenberg et al. 2015c]. The physiological characterization of strain LHL191014123 with the Omnilog GEN III plate identification system did not reveal a superior resolution compared to other standard tests, although a panel 
of 94 different reactions was assessed. Even the most sensitive protocols for fastidious bacteria (C1 [24 h] and $\mathrm{C} 2$ [48 $\mathrm{h}$ incubation]) with and without addition of $10 \%$ bovine serum resulted in very weak reactions. Slightly positive reactions were found only for Laspartic acid, L-glutamic acid, D-glucuronic acid, glucuronamide, L-lactic acid, citric acid, $\alpha$-ketobutyric acid, sodium butyrate and sodium bromate (data not shown).

\section{MALDI-TOF MS}

MALDI-TOF MS spectra of strain LHL191014123 show the $\mathrm{m} / \mathrm{z}$ signals typical of Streptobacillus genus at 3,631.2 $\pm 3.6,7,262.0 \pm 7.3$, and 7,392.0 \pm 7.4 (data not shown, Eisenberg et al. 2018). Nevertheless, the strain LHL191014123 had score values lower than 1.5 with the used commercial Bruker database version and was, therefore, not identified. The application of the enlarged database, extended by reference entries for each of the known members of the genus Streptobacillus, including the type strain of $S$. felis $131000547^{\mathrm{T}}$, allowed the unequivocal assignment of strain LHL191014123 to S. felis (score values up to 2.719). Custom-made MSP of all Streptobacillus species were prepared from known type strains based on Bruker quality criteria and used in this study; respective MSP are available for exchange via the MALDI-user platform MALDI-UP (https://maldi-up. ua-bw.de) (Rau et al. 2016). A dendrogram depicting the topologic position of the reference spectrum of strain LHL191014123 from the rusty-spotted cat from this study to closely related strains of the other known streptobacilli and related taxa is shown in Fig. 2.

\section{Molecular characterization}

PCR analysis

Both earlier published PCR protocols for the detection of S. moniliformis are based on the Streptobacillus $16 \mathrm{~S}$ rRNA gene. As expected, strain LHL191014123 from this study gave a positive amplification in these two PCR assays. The recently designed gyrB gene PCR was also positive for strain LHL191014123.

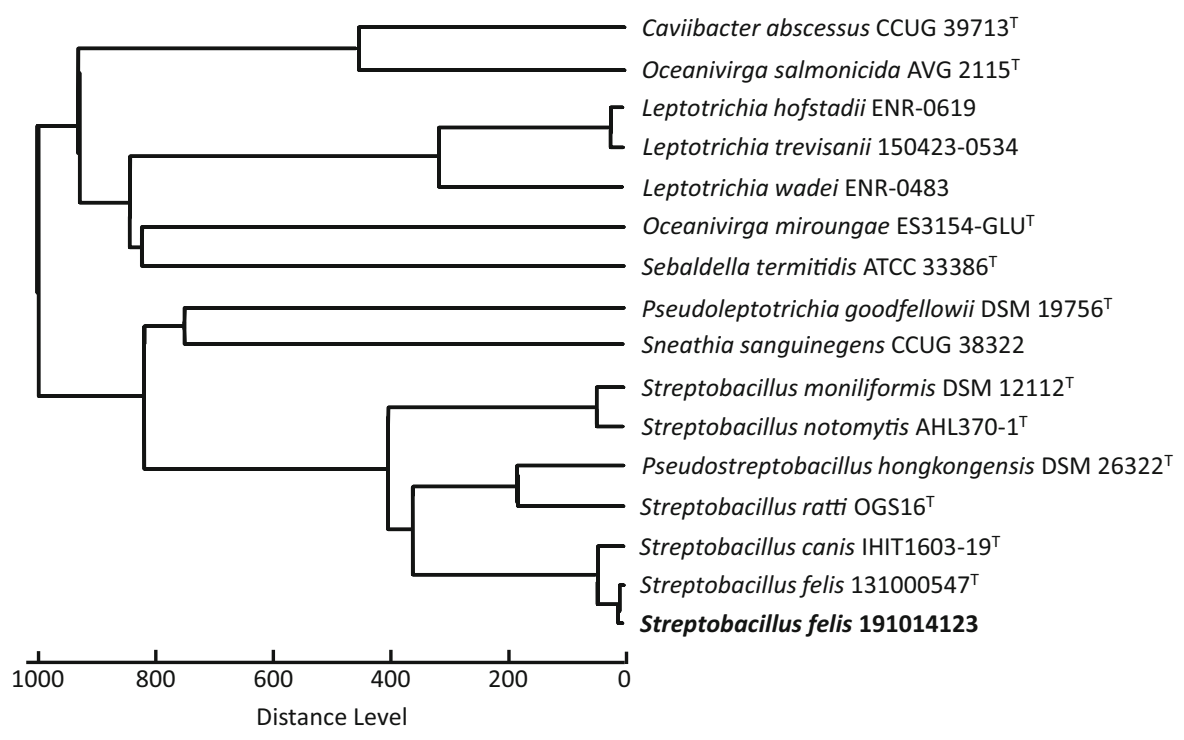

Fig. 2 Dendrogram including reference main spectra (MSP) of the family Leptotrichiaceae available in the Bruker Taxonomy Database; spectra of Streptobacillus canis IHIT1603-19 ${ }^{\mathrm{T}}$, S. felis $131000547^{\mathrm{T}}$, S. notomytis AHL $370-1^{\mathrm{T}}$, S. ratti OGS $16^{\mathrm{T}}$, Pseudostreptobacillus hongkongensis DSM $26322^{\mathrm{T}}$, Caviibacter abscessus CCUG39713 ${ }^{\mathrm{T}}$, Oceanivirga salmonicida AVG2115 ${ }^{\mathrm{T}}$, Oceanivirga miroungae ES3154-GLU ${ }^{\mathrm{T}}$, Sebaldella termitidis $\mathrm{NCTC}_{11300}{ }^{\mathrm{T}}$, Sneathia sanguinegens CCUG41628 ${ }^{\mathrm{T}}$ reference strains were recorded using an acetonitrile-formic acid extraction protocol. The dendrogram was generated using the MBT Compass Explorer MSP Dendrogram Creation Standard Method (v1.4) of the MALDI Biotyper OC Software (v3.1, build 66). The database used (DB 8,468, BrukerDaltonics) comprised only strains of Streptobacillus moniliformis including the the type strain DSM $12112^{\mathrm{T}}$ as well as spectra of the depicted Leptotrichia spp.; ${ }^{\mathrm{T}}$, type strain; ENR, European Network for the Rapid Identification of Anaerobes (ENRIA) 
Genomic features

The draft genome $(1,386,907 \mathrm{bp})$ consists of 163 contigs and possesses 1,345 CDS, 2 rRNA and 38 tRNA. Analysis of further genomic features revealed one prophage (PHAGE_Gordon_Smoothie_NC_030696, (Zhou et al. 2011)) and 96 tandem repeats (Benson 1999). However, screening for CRISPR regions was negative in contrast to $S$. felis type strain $\left(131000547^{\mathrm{T}}\right)$ that was found to possess a relatively large CRISPR region $(2,078 \mathrm{bp})$ with 31 spacers (Grissa et al. 2007). Five genomic islands with a size range 3,220 to 66,556 bp were also identified (Bertelli et al. 2017). Four islands possess mainly hypothetical proteins and none seems to express pathogenic factors. However, one island (26,565 bp) seems to be associated with transport and metabolism of different substrates including carbohydrates and minerals. Interestingly, genome analysis with Pathogenfinder (Cosentino et al. 2013) suggested that S. felis (LHL191014123 as well as $131000547^{\mathrm{T}}$ ) has a probability of 0.976 to be a human pathogen since it harbors seven pathogenic families, all of which originate from $S$. moniliformis, the classical pathogen of the RBF zoonosis. This web-tool predicts the pathogenicity of a submitted genome based on a model that compares its sequence data to a protein family database containing proteins known to be associated with pathogenic or non-pathogenic bacteria.

Phylogenetic and phylogenomic analyses

The 16S rRNA gene sequence of strain LHL191014123 was derived from WGS and represents a stretch of 1,514 unambiguous nucleotides. This sequence was blasted against the quality-controlled database EzBioCloud (Yoon et al. 2017) and highest similarities to the type strains of S. felis (99.93\%), $S$. canis $(98.68 \%)$, S. notomytis $(98.26 \%)$, S. ratti (97.85\%), S. moniliformis (97.64\%) and P. hongkongensis $(94.23 \%)$, followed by Oceanivirga salmonicida $(91.10 \%)$ and 'Sneathia amnii' $(90.58 \%)$ were found. In a $16 \mathrm{~S}$ rRNA gene sequence phylogenetic tree (ML algorithm), strain LHL191014123 clustered most closely and in a separate branch together with the type strain of $S$. felis. The next closely related species was $S$. canis that grouped as a sister clade to $S$. felis with high bootstrap support (data not shown). Based on partial nucleotide sequences of the groEL, gyrB
(Suppl. Fig. S1) and recA genes, this topologic position was also identical for the investigated housekeeping genes. A core genome phylogeny of strain LHL191014123 and 20 genomes of the family Leptotrichiaceae was calculated in EDGAR 2.3 based on MUSCLE alignment as previously described (Eisenberg et al. 2017b). This resulted in one multiple alignment of 267 core genes per genome (5,607 genes in total), with 95,146 amino acid residues per genome (1,998,066 in total). The Neighbor-Joining algorithm (Fig. 3) as well as the approximately-Maximum Likelihood phylogeny (data not shown) both confirmed the taxonomic position of strain LHL191014123 as a member of Streptobacillus felis with $S$. canis being the closest relative, still with a larger phylogenetic distance. Species identity between the rusty-spotted cat's strain to $S$. felis was once more confirmed by mean average nucleotide identity (ANI) values of $99.32 \%$ (reciprocal 99.24), which is clearly above the $>95-96 \%$ proposed boundary for identical species (Goris et al. 2007).

Accession numbers and strain deposition

The GenBank/ENA/DDBJ accession numbers for the 16S rRNA, groEL, gyrB and recA gene sequences of strain LHL191014123 as well as for the complete genome sequence are MN764137, MN793979, MN793980, MN793981 and genome acc. no. (JABMKT000000000; BioSample SAMN14996772; BioProject PRJNA634464), respectively. Further accession numbers of $g y r B$ sequences from mouth swabs from cats are MT498840-MT498846 and have been published in Matt et al. (2020). Strain LHL191014123 has been deposited at the German Collection of Microorganisms and Cell Cultures (DSMZ), the Culture Collection of the University Gotenburg (CCUG), the Collection of Institute Pasteur (CIP) and the strain collection of the Hessian State Laboratory (LHL) under identifiers DSM110500, CCUG74119, CIP111794 and LHL191014123.

\section{Discussion}

It is evident from the depicted molecular results and from MALDI-TOF MS analysis that LHL191014123 is an additional strain of S. felis. To our knowledge, this is the second available strain with proper species 


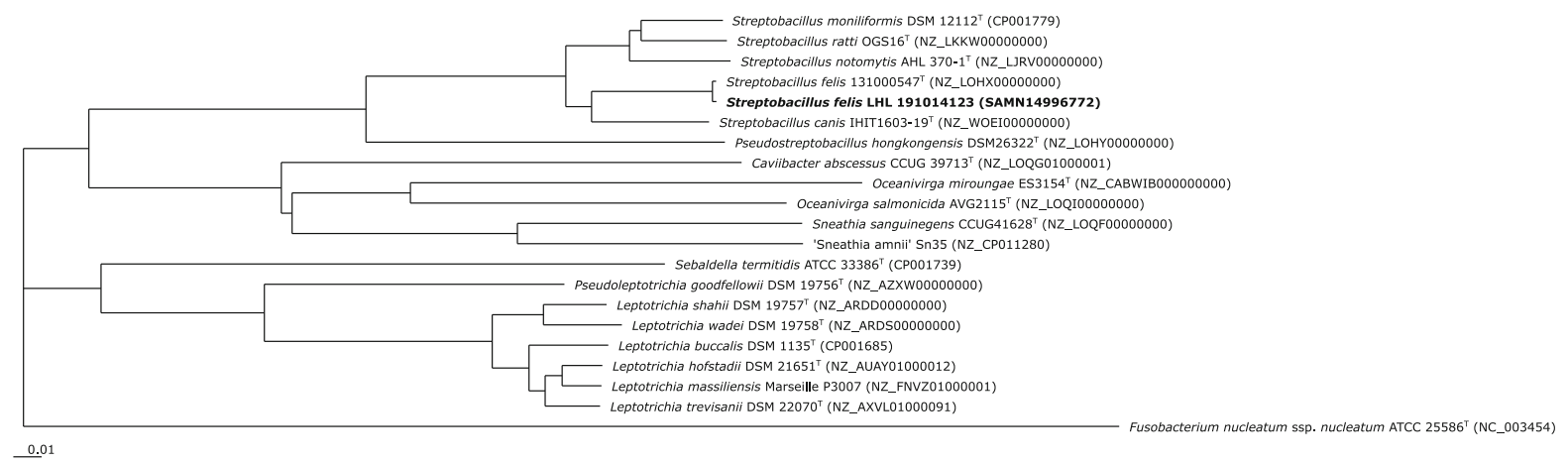

Fig. 3 Core genome phylogenetic tree depicting strain LHL191014123 within the family Leptotrichiaceae. Core genes of these genomes were computed in EDGAR 2.3 based on MUSCLE alignments and the Neighbor-Joining algorithm as implemented in the PHYLIP package. The core genome analysis was based on of 267 genes per genome in 17 type species genomes (5,607 in total) of the family Leptotrichiaceae. The core has 95,146 amino acid residues and 1,998,066 bp per

identification and an extended genetic and phenotypic knowledge base. Although we could recently show that approximately $50 \%$ of randomly selected, mostly healthy domestic cats harbor S. felis (Matt et al. 2020), the isolation of these streptobacilli from cats is a rare exceptional case. A number of studies have indicated dogs and cats as possible vectors of $S$. moniliformis to humans, especially after mouthing wild rats (Gascard et al. 1967; Maynard et al. 1986; Mollaret 1969; Peel 1993; Wouters et al. 2008). However, isolates have not been stored and phylotypes are not available for most of the mentioned studies. Therefore, these microorganisms cannot be verified as $S$. moniliformis. Conversely, 16S rRNA gene phylotypes from one former and additional studies in dogs suggested a much closer relationship of their Streptobacillus OTUs to $S$. canis than to S. moniliformis (Dewhirst et al. 2012; Xenoulis et al. 2008) (Fig. 1). Relatively few dogs have suffered from streptobacillosis (Das 1986; Ditchfield et al. 1961).

OTU sequences of cats that are most closely related to $S$. felis were previously lacking, but have recently been found in half of the investigated cats (Matt et al. 2020) and also closely related bacterial species (uncultured 'Leptotrichia', 'Leptotrichiaceae') have been detected at various body sites (Older et al. 2019; Sturgeon et al. 2014). One study mentions two Streptobacillus isolates but without any further identification or disease association (Whyte et al. 2017). genome in total. GenBank accession numbers are given in parentheses. "Sneathia amnii" and "Leptotrichia massiliensis" were included, however, these taxonomic names have been effectively published but not validly published under the rules of the International Code of Nomenclature of Bacteria. Fusobacterium nucleatum is used as outgroup. "T" indicating type strain; Bar, 0.01 amino acid substitutions per site

The species S. felis has been described from cats as well as from a human patient with contact to cats, suggesting that this microorganism may be a member of the cats' microbiota with the potential to cause zoonotic infections (Eisenberg et al. 2015a; Matt et al. 2020). The high occurrence (50\% found in Matt et al. (2020)) should be considered with respect to the potential role of $S$. felis both as a cat pathogen and a potentially zoonotic microorganism because cats represent the most popular pet animal species and streptobacillosis is considered a significantly underreported disease. However, possible reasons why streptobacilli have been infrequently diagnosed may include a lack of awareness of the disease among clinicians, an absence of pathognomic signs of disease in animals, a lack of reliable diagnostics, fastidious growth of the pathogen, susceptibility to most antibiotics used for empiric therapy, unnoticed animal contact and that this is a non-notifiable disease worldwide (Eisenberg 2017).

S. felis has first been isolated from a cat with acute bronchopneumonia and a myocardium with multifocal haemorrhages on the endo- and epicardium (Eisenberg et al. 2014). In this second report, again lungs were predominantly affected, but streptobacilli were isolated from all major organs pointing towards an agonal spread of these bacteria or an early septicemia. Likewise, the next closely related species, S. canis, has recently been found to also constitute a member of 
canine oral microbiota (Matt et al. 2020) and has been isolated from a phlegmon on a dog's hindleg (Eisenberg et al. 2020b). Hypothesizing that bite wounds are often caused by oral microbiota (Abrahamian and Goldstein 2011), one can speculate that streptobacilli from cats might occasionally also be involved in wound infections.

Interestingly, a novel diagnostic immune-histology tool for the detection of $S$. moniliformis turned out to reveal negative results throughout. The method was found to successfully detect experimentally infected mice (data not shown). Because the lack of suitable diagnostics for the detection of streptobacillosis is often referred to as a diagnostic dilemma (Mahmoodi et al. 2016; Rumley et al. 1987), the novel IHC assay represents a promising diagnostic tool to improve this situation and to identify $S$. moniliformis in situ. The lack of binding in the here presented case suggests deviant epitopes in S. felis.

\section{Conclusion}

This is the second isolation of $S$. felis in a diseased cat species. A preliminary sampling of cats revealed that this microorganism is frequently found in the oropharynx and that cats represent a reservoir for S. felis. However, this is further evidence that this species is cat specific but with a broader distribution in feline hosts than previously thought. Further studies are necessary to elucidate the role of $S$. felis in domestic and other cat species in order to better estimate its zoonotic potential.

Acknowledgements Open Access funding provided by Projekt DEAL. We like to thank all cat owners for their participation in the prevalence assessment. For excellent technical assistance, we thank Mersiha Curic, Matrin Dyk, Katharina Engel, Jens Heinbächer, Ulrike Kling and MarieLuise Sonneborn. The Hessian Ministry for the Environment, Climate Change, Agriculture and Consumer Protection (HMUKLV) supports the Hessian State Laboratory.

Authors' contributions AF and TE designed the microbiological study. NS and CG were responsible for animal care. TE isolated the $S$. felis strain. KR conducted gross pathology and histology; $\mathrm{AB}$ and $\mathrm{CH}$ were responsible for IHC. JR was in charge of the MALDI-TOF MS analysis. JF and CI performed the WGS, JB and AF were involved in data analyses. TE and AF interpreted the data and wrote the manuscript. All authors critically checked and contributed to the final version of the manuscript.
Funding This research received no specific grant from any funding agency in the public, commercial, or not-for-profit sectors. The Hessian Ministry for the Environment, Climate Change, Agriculture and Consumer Protection supports the Hessian State Laboratory.

Availability of data and material All data have been made fully available to the public.

\section{Compliance with ethical standards}

Conflicts of interest Not applicable.

Ethics approval There are no ethical issues associated with this manuscript. Animal husbandry fulfilled ethical standard guidelines according to the code of ethics and animal welfare of the World Association of Zoos and Aquariums (WAZA; https:// www.waza.org/priorities/animal-welfare/). Sampling of the rusty-spotted cat followed veterinary euthanasia of the diseased animal.

Consent to participate All authors gave their consent to participate in this study.

Consent for publication All authors gave their consent to publish results from this study and to be listed as a co-author.

Open Access This article is licensed under a Creative Commons Attribution 4.0 International License, which permits use, sharing, adaptation, distribution and reproduction in any medium or format, as long as you give appropriate credit to the original author(s) and the source, provide a link to the Creative Commons licence, and indicate if changes were made. The images or other third party material in this article are included in the article's Creative Commons licence, unless indicated otherwise in a credit line to the material. If material is not included in the article's Creative Commons licence and your intended use is not permitted by statutory regulation or exceeds the permitted use, you will need to obtain permission directly from the copyright holder. To view a copy of this licence, visit http://creativecommons.org/licenses/by/4.0/.

\section{References}

Abrahamian FM, Goldstein EJ (2011) Microbiology of animal bite wound infections. Clin Microbiol Rev 24:231-246. https://doi.org/10.1128/CMR.00041-10

Benson G (1999) Tandem repeats finder: a program to analyze DNA sequences. Nucleic Acids Res 27:573-580. https:// doi.org/10.1093/nar/27.2.573

Bertelli C, Laird MR, Williams KP, Simon Fraser University Research Computing G, Lau BY, Hoad G, Winsor GL, Brinkman FSL (2017) IslandViewer 4: expanded prediction of genomic islands for larger-scale datasets. Nucleic Acids Res 45:30-35. https://doi.org/10.1093/nar/gkx343

Blom J, Kreis J, Spanig S, Juhre T, Bertelli C, Ernst C, Goesmann A (2016) EDGAR 2.0: an enhanced software 
platform for comparative gene content analyses. Nucleic Acids Res 44:22-28. https://doi.org/10.1093/nar/gkw255

Cosentino S, Voldby Larsen M, Moller Aarestrup F, Lund O (2013) PathogenFinder-distinguishing friend from foe using bacterial whole genome sequence data. PLoS ONE 8:e77302. https://doi.org/10.1371/journal.pone.0077302

Das AM (1986) Streptobacillus moniliformis isolated from an abcess of a dog. Ind J Comp Microbiol Immunol Infect Dis $7: 115$

Dewhirst FE, Klein EA, Thompson EC, Blanton JM, Chen T, Milella L, Buckley CM, Davis IJ, Bennett ML, MarshallJones ZV (2012) The canine oral microbiome. PLoS ONE 7:e36067. https://doi.org/10.1371/journal.pone.0036067

Ditchfield J, Lord LH, McKay KA (1961) Streptobacillus moniliformis infection in a dog. Can Vet J 2:457-459

Eisenberg T (2017) Phylogenetic investigations and comparative genome analyses within the family Leptotrichiaceae with special consideration of Streptobacillus moniliformis, the causative organism of rat bite fever. Institute for Hygiene and Infectious Diseases of animals (Habilitation Thesis). Justus-Liebig-University, Giessen

Eisenberg T, Nesseler A, Nicklas W, Spamer V, Seeger H, Zschöck M (2014) Streptobacillus sp. isolated from a cat with pneumonia. J Clin Microbiol Case Rep 2014:1-7. https://doi.org/10.1099/jmmcr.0.000562

Eisenberg T, Glaeser S, Nicklas W, Mauder N, Contzen M, Aledelbi K, Kämpfer P (2015a) Streptobacillus felis sp. nov. isolated from a cat with pneumonia. Int J Syst Evol Microbiol 65:2172-2178. https://doi.org/10.1099/ijs.0. 000238

Eisenberg T, Glaeser SP, Ewers C, Semmler T, Nicklas W, Rau J, Mauder N, Hofmann N, Imaoka K, Kimura M, Kämpfer P (2015b) Streptobacillus notomytis sp. nov. isolated from a spinifex hopping mouse (Notomys alexis THOMAS, 1922), and emended description of Streptobacillus Levaditi et al. 1925, Eisenberg et al. 2015 emend. Int J Syst Evol Microbiol 65:4823-4829. https://doi.org/10.1099/ijsem.0. 000654

Eisenberg T, Nicklas W, Mauder N, Rau J, Contzen M, Semmler T, Hofmann N, Aledelbi K, Ewers C (2015c) Phenotypic and genotypic characteristics of members of the genus Streptobacillus. PLoS ONE 10:e0134312. https://doi.org/ 10.1371/journal.pone.0134312

Eisenberg T, Imaoka K, Kimura M, Glaeser SP, Ewers C, Semmler T, Rau J, Nicklas W, Kämpfer P (2016) Streptobacillus ratti sp. nov., isolated from a black rat (Rattus rattus). Int J Syst Evol Microbiol 66:1620-1626. https:// doi.org/10.1099/ijsem.0.000869

Eisenberg T, Poignant S, Jouan Y, Fawzy A, Nicklas W, Ewers C, Mereghetti L, Guillon A (2017a) Acute tetraplegia caused by rat bite fever in snake keeper and transmission of Streptobacillus moniliformis. Emerg Infect Dis 23:719-721. https://doi.org/10.3201/eid2304.161987

Eisenberg T, Riße K, Schauerte N, Geiger C, Blom J, Scholz HC (2017b) Isolation of a novel 'atypical' Brucella strain from a bluespotted ribbontail ray (Taeniura lymma). Antonie Van Leeuwenhoek 110:221-234. https://doi.org/10.1007/ s10482-016-0792-4

Eisenberg T, Glaeser SP, Blom J, Rau J, Kämpfer P (2018) Genus Streptobacillus. In: Whitman WB (ed) Bergey's manual of systematics of archaea and bacteria. Wiley, Hoboken, pp 1-7

Eisenberg T, Glaeser SP, Blom J, Kämpfer P (2020a) Proposal to reclassify Streptobacillus hongkongensis into a novel genus as Pseudostreptobacillus hongkongensis gen. nov., comb. nov. IJSEM in press https://doi.org/10.1099/ijsem.0. 004051

Eisenberg T, Heydel C, Prenger-Berninghoff E, Fawzy A, Kling U, Akimkin V, Semmler T, Mühldorfer K, Kämpfer P, Blom J, Ewers C (2020b) Streptobacillus canis sp. nov. isolated from a dog. Int J Syst Evol Microbiol. https://doi. org/10.1099/ijsem.0.004086

Fawzy A, Zschöck M, Ewers C, Eisenberg T (2016) New polymorphisms within the variable number tandem repeat (VNTR) 7 locus of Mycobacterium avium subsp. paratuberculosis. Mol Cell Probes 30:132-137. https://doi.org/ 10.1016/j.mcp.2016.02.002

Fornefett J, Krause J, Klose K, Fingas F, Hassert R, Eisenberg T, Schrödl W, Grunwald T, Müller U, Baums CG (2017) Comparative analysis of clinics, pathologies and immune responses in BALB/c and C57BL/6 J mice infected with Streptobacillus moniliformis. Microbes Infect. https://doi. org/10.1016/j.micinf.2017.10.001

Fukushima K, Yanagisawa N, Imaoka K, Kimura M, Imamura A (2017) Rat-bite fever due to Streptobacillus notomytis isolated from a human specimen. J Infect Chemother Off J Jpn Soc Chemother. https://doi.org/10.1016/j.jiac.2017.10. 018

Gaastra W, Boot R, Ho HT, Lipman LJ (2009) Rat bite fever. Vet Microbiol 133:211-228. https://doi.org/10.1016/j. vetmic.2008.09.079

Gascard E, Vignoli R, Moulard JC, Salvadori JM (1967) Case of febrile eruption after a cat bite: streptobacillus moniliformis septicemia? Mars Med 104:861-864

Goris J, Konstantinidis KT, Klappenbach JA, Coenye T, Vandamme P, Tiedje JM (2007) DNA-DNA hybridization values and their relationship to whole-genome sequence similarities. Int J Syst Evol Microbiol 57:81-91. https:// doi.org/10.1099/ijs.0.64483-0

Grissa I, Vergnaud G, Pourcel C (2007) CRISPRFinder: a web tool to identify clustered regularly interspaced short palindromic repeats. Nucleic Acids Res 35:52-57. https:// doi.org/10.1093/nar/gkm360

Kearse M, Moir R, Wilson A, Stones-Havas S, Cheung M, Sturrock S, Buxton S, Cooper A, Markowitz S, Duran C, Thierer T, Ashton B, Meintjes P, Drummond A (2012) Geneious Basic: an integrated and extendable desktop software platform for the organization and analysis of sequence data. Bioinformatics 28:1647-1649. https://doi. org/10.1093/bioinformatics/bts199

Kimura M, Tanikawa T, Suzuki M, Koizumi N, Kamiyama T, Imaoka K, Yamada A (2008) Detection of Streptobacillus spp. in feral rats by specific polymerase chain reaction. Microbiol Immunol 52:9-15. https://doi.org/10.1111/j. 1348-0421.2008.00005.x

Lau SK, Chan JF, Tsang CC, Chan SM, Ho ML, Que TL, Lau YL, Woo PC (2016) Human oropharynx as natural reservoir of Streptobacillus hongkongensis. Sci Rep 6:24419. https://doi.org/10.1038/srep24419

Levaditi C, Nicolau S, Poincloux P (1925) Sur le rôle étiologique de Streptobacillus moniliformis (nov. spec.) dans 
l'érythème polymorphe aigu septicémique. C R Acad Sci 180:1188-1190

Mahmoodi E, Grainge C, Erdstein A, O’kane G (2016) Septic arthritis caused by pet rodents: a diagnostic dilemma. Australas Med J 9:270-273

Matt U, Schmiedel J, Fawzy A, Trauth J, Schmidt K, Vogel K, Herold S, Karrasch T, Imirzalioglu C, Eisenberg T (2020) Infection in a young immunocompetent male caused by Streptobacillus felis, a putative zoonotic microorganism transmitted by cats. Clin Infect Dis Press. https://doi.org/ $10.1093 / \mathrm{cid} / \mathrm{ciaa} 968$

Maynard JH, McNaughton WM, Travis T (1986) Streptobacillus moniliformis cellulitis and bacteraemia following a dog bite. Commun Dis Intell 1:10

Mollaret P (1969) Infections following cat and rat bites. Munch Med Wochenschr 111:13-18

Ogawa Y, Kasahara K, Lee ST, Ito T, Hasegawa H, Hirose S, Santo S, Yoshida A, Nakano R, Yano H, Mikasa K (2018) Rat-bite fever in human with Streptobacillus notomytis infection, Japan. Emerg Infect Dis 24:1377-1379. https:// doi.org/10.3201/eid2407.171580

Older CE, Diesel AB, Lawhon SD, Queiroz CRR, Henker LC, Rodrigues Hoffmann A (2019) The feline cutaneous and oral microbiota are influenced by breed and environment. PLoS ONE 14:e0220463. https://doi.org/10.1371/journal. pone. 0220463

Peel MM (1993) Dog-associated bacterial infections in humans: isolates submitted to an Australian reference laboratory, 1981-1992. Pathol 25:379-384

Rau J, Eisenberg T, Männig A, Wind C, Lasch P, Sting R (2016) MALDI-UP-An internet platform for the exchange of MALDI-TOF mass spectra. User guide for http://maldi-up. ua-bw.de/. Aspects of food control and animal health (eJournal) 2016:1-17

Richter M, Rossello-Mora R (2009) Shifting the genomic gold standard for the prokaryotic species definition. Proc Natl Acad Sci USA 106:19126-19131. https://doi.org/10.1073/ pnas.0906412106

Rohde J, Rapsch C, Fehr M (2008) Case report: abscessation due to Streptobacillus moniliformis in a rat [in German]. Prakt Tierarzt 89:466-473
Rumley RL, Patrone NA, White L (1987) Rat-bite fever as a cause of septic arthritis: a diagnostic dilemma. Ann Rheum Dis 46:793-795

Sturgeon A, Pinder SL, Costa MC, Weese JS (2014) Characterization of the oral microbiota of healthy cats using nextgeneration sequencing. Vet J 201:223-229. https://doi.org/ 10.1016/j.tvj1.2014.01.024

Whyte A, Gracia A, Bonastre C, Tejedor MT, Whyte J, Monteagudo LV, Simon C (2017) Oral disease and microbiota in free-roaming cats. Top Companion Anim Med 32:91-95. https://doi.org/10.1053/j.tcam.2017.07.003

Woo PC, Wu AK, Tsang CC, Leung KW, Ngan AH, Curreem SO, Lam KW, Chen JH, Chan JF, Lau SK (2014) Streptobacillus hongkongensis sp. nov., isolated from patients with quinsy and septic arthritis, and emended descriptions of the genus Streptobacillus and the species Streptobacillus moniliformis. Int J Syst Evol Microbiol 64:3034-3039. https://doi.org/10.1099/ijs.0.061242-0

Wouters EG, Ho HT, Lipman LJ, Gaastra W (2008) Dogs as vectors of Streptobacillus moniliformis infection? Vet Microbiol 128:419-422. https://doi.org/10.1016/j.vetmic. 2007.10.019

Xenoulis PG, Palculict B, Allenspach K, Steiner JM, Van House AM, Suchodolski JS (2008) Molecular-phylogenetic characterization of microbial communities imbalances in the small intestine of dogs with inflammatory bowel disease. FEMS Microbiol Ecol 66:579-589. https://doi.org/ 10.1111/j.1574-6941.2008.00556.x

Yoon SH, Ha SM, Kwon S, Lim J, Kim Y, Seo H, Chun J (2017) Introducing EzBioCloud: a taxonomically united database of 16S rRNA gene sequences and whole-genome assemblies. Int J Syst Evol Microbiol 67:1613-1617. https://doi. org/10.1099/ijsem.0.001755

Zhou Y, Liang Y, Lynch KH, Dennis JJ, Wishart DS (2011) PHAST: a fast phage search tool. Nucleic Acids Res 39:347-352. https://doi.org/10.1093/nar/gkr485

Publisher's Note Springer Nature remains neutral with regard to jurisdictional claims in published maps and institutional affiliations. 